\title{
A neural network approach to option pricing
}

\author{
F. Mostafa \& T. Dillon \\ DEB1 Institute, Curtin University, Australia
}

\begin{abstract}
In this paper the pricing performance of the artificial neural network is compared to the Black-Scholes and the GARCH option-pricing model. The artificial neural network is trained on the implied volatility rather then the option price, which leads to an improved performance when compared to the competing models. The hedging performance of the neural network, GARCH option-pricing model and the Black-Scholes are also analysed.

Keywords: neural networks, option pricing, hedging, implied volatility, GARCH option pricing model.
\end{abstract}

\section{Introduction}

Since the publication of the Black-Scholes model in 1973 (Black and Scholes [1]), it remains the most quoted scientific paper in the world. The model made a key contribution to option trading, where investors are able to calculate a fair value of an option contract. This model had its limitations, which stem from the unrealistic assumptions. The Black-Scholes behaviour has been well documented in literature (Henderson [2]). The most interesting stylised fact of the BlackScholes model that has captured the attention of researches and practitioners alike is the volatility skew (or volatility smile). This stylised fact can be seen when the implied volatility is backed out from the Black-Scholes formula and is plotted with respect to the option moneyness. The graph deviated from a flat line, which is a contradiction to the constant volatility assumption of the BlackScholes.

Researchers then turned to more sophisticated methods for option valuation using stochastic volatility models (Ritchken and Trevor [3], Peter and Kris [4], Engle and Mustafa [5], Duan [6] and Heston et al. [7]). The GARCH option pricing model (GOPM) introduced by Duan [6] is based on a discrete-time 
model of the economy. The GOPM is derived based on the locally riskneutralised probability measure where the option value can be calculated as a discounted expected value. In addition, the model allows the underlying asset return to follow a GARCH process (Bollerslev [8]). Consequently, eliminating the assumption of a constant volatility. This is indeed an attractive feature for the GOPM, however the main drawback of the model is that it does not have a closed form solution. Therefore, Monte Carlo simulation is used to optimise the models parameters. Duan and Simonato [9] have introduced empirical martingale simulation that speeds up the Monte Carlo simulation process.

Recently research has also adopted different methods for pricing options, such as neural networks (Karaali et al. [10]). The vast majority of neural networks research has been focused on forecasting financial time series (Schittenkopf et al. [11], Kaastra and Boyd [12]). Until the late 1990's research on option pricing with neural networks was limited. To date most research compares the performance of neural networks to the Black-Scholes optionpricing model (Meissner and Kawano [13], Amilon [14], Yao et al. [15], Tino et al. [16] and Bennell and Sutcliffe [17]). Most research demonstrates comparable or slightly better performance of neural networks to the tradition models. However, there has been no real attempt to compare the neural networks to more advanced models such as the GARCH option pricing model (GOPM). In Hanke [18], a neural network was constructed to give an approximate price to GOPM. This was achieved by training the network on different input combinations. Thus, utilising the network output as an approximation to the option pricing formula to overcoming the numerical simulation issues.

The aim of this paper is improve on the neural networks ability to price option out-of-sample by learning the Black-Scholes implied volatility. The implied volatility produced by the neural networks is then used in the Black-Scholes formula to produce the neural network valuation of the option. The performance of the neural networks is then compared to the GARCH Option pricing model and the Black-Scholes based on the pricing and hedging criteria. Section two of this paper examines the competing models. The historical data used in this research is described in section three. The experiential design is illustrated in section four. Sections five and six describe the methods used for the pricing errors and the results achieved. The summary and conclusion are discussed in section seven.

\section{Competing models}

In this research, we study the out of sample pricing capabilities of different option pricing models. The GARCH option pricing model and the Black-Scholes are compared with two types of neural network designs. The first design is the common approach using the option price as the target output of the network. The second method is to train the neural network directly on the Black-Scholes implied volatility and then using the Black-Scholes formula to derive the theoretical price. 


\subsection{GARCH Option Pricing Model (GOPM)}

The GARCH Option Pricing Model (GOPM) introduced by Duan [6] is based on a discrete-time model of the economy. The value of the index at time $t$, can be assumed to have the following dynamics,

$$
\begin{gathered}
r_{t}=r_{f}+\lambda \sigma_{t}-\frac{1}{2} \sigma_{t}+\sigma_{t} \varepsilon_{t} \\
\varepsilon_{t} \mid \Omega_{t-1} \sim N(0,1) \\
\sigma_{t}^{2}=\beta_{0}+\beta_{1}\left(\varepsilon_{t-1}-\gamma\right)^{2} \sigma_{t-1}^{2}+\beta_{2} \sigma_{t-1}^{2}
\end{gathered}
$$

Where $\lambda$ is interpreted as the risk premium. To ensure the variance is positive and stationary the following constraints are applied:

$$
\begin{aligned}
& \beta_{0}>0 \\
& \beta_{1} \geq 0 \\
& B_{2} \geq 0 \\
& \beta_{1}\left(1+\lambda^{2}\right)+\beta_{2}<1
\end{aligned}
$$

The unconditional variance is given by $\beta_{0} /\left(1-\beta_{1}\left(1+\lambda^{2}\right)-\beta_{2}\right)$. This process is reduced to the Black-Scholes homoskedastic lognormal process when $\beta_{1}=0$ and $\beta_{2}=0$.

It has been demonstrated by Duan [6] that under the Local Risk Neutral Valuation Relationship (LRNVR) the conditional variance does not change , however under measure $Q$ the conditional expectation of $\mathrm{r}_{\mathrm{t}}$ is the risk free rate $\mathrm{r}_{\mathrm{f}}$.

$$
E^{Q}\left[\exp \left(r_{t}\right) \mid \Omega_{t-1}\right]=\exp \left(r_{f}\right)
$$

To derive the GOPM, the risk neutral valuation relationship has to be generalized to the LRNVR:

$$
\begin{aligned}
r_{t} & =r_{f}-\frac{1}{2} \sigma_{t}^{* 2}+\sigma_{t}^{*} \varepsilon_{t}^{*}, \\
\varepsilon_{t}^{*} & \sim N(0,1), \\
\sigma_{t}^{* 2} & =\beta_{0}+\beta_{1}\left(\varepsilon_{t-1}^{*}-\tilde{\gamma}\right)^{2} \sigma_{t-1}^{* 2}+\beta_{2} \sigma_{t-1}^{* 2}
\end{aligned}
$$

By having $\tilde{\gamma}=\lambda+\gamma$, the risk-neutral pricing measure is determined by four parameters, $\beta_{0}, \beta_{1}, \beta_{2}$ and $\gamma$. Using the above formulation the asset terminal is then calculated at time $\mathrm{T}$

$$
S_{T}=S_{t} \exp \left(r_{f}(T-t)-\frac{1}{2} \sum_{i=t+1}^{T} \sigma_{i}^{* 2}+\sum_{i=t+1}^{T} \sigma_{i}^{*} \varepsilon_{i}^{*}\right)
$$


The terminal asset price is then calculated using Monte Carlo Simulation. A set of $\mathrm{N}$ random path of residuals $\left(\varepsilon_{t+1, j}^{*}, \ldots, \varepsilon_{T, j}^{*}\right)$ are generated with $\mathrm{J}=1$ to $\mathrm{N}$. The residuals are used to calculate the asset prices $\mathrm{S}_{\mathrm{T}, \mathrm{j}}$. Using the terminal asset price series the option price is then obtained by risk-neutral conditional expectation $\mathrm{E}^{*}$ :

$$
C_{\text {GARCH }}=\exp \left(-r_{f}^{*}(T-t)\right) E^{*}\left[\max \left(S_{T}-K, 0\right)\right]
$$

The final option price is then approximated as follow:

$$
C_{\text {GARCH }}=\exp \left(-r_{f}^{*}(T-t)\right) \frac{1}{N} \sum_{j=1}^{M} \max \left(S_{T, j}-K, 0\right)
$$

Rather than using standard Monte Carlo simulation, Empirical Martingale Simulation (EMS) is adopted. The EMS method has been shown to accelerate the convergence of the Monte Carlo prices estimates as demonstrated by Duan and Simonato [9].

The Monte Carlo simulation is give by:

$$
\begin{aligned}
& S_{t}(i)=S_{0} \exp \left[r_{f} t\right] Z_{t}(i) \\
& Z_{t}(i)=Z_{t-1}(I) \exp \left[-0.5 \sigma_{t}^{2}+\sigma_{t} \varepsilon_{t}(i)\right]
\end{aligned}
$$

and for the EMS, $Z_{t}(i)=\frac{Z_{t-1}(i) \exp \left[-0.5 \sigma_{t}^{2}+\sigma_{t} \varepsilon_{t}(i)\right]}{\frac{1}{n} \sum_{i=1}^{n} Z_{t-1}(i) \exp \left[-0.5 \sigma_{t}^{2}+\sigma_{t} \varepsilon_{t}(i)\right]}$

The delta that corresponds to the GOPM is given by Duan [6]:

$$
\Delta_{t}^{G}=\exp \{-(T-t) r\} E\left[\frac{S_{T}}{S_{t}} I\left(S_{T}, K\right) \mid \phi_{t}\right]
$$

Where $\mathrm{I}\left(\mathrm{S}_{\mathrm{t}}, \mathrm{X}\right)=1$ is $\mathrm{S}_{\mathrm{t}} \geq \mathrm{K}$ and 0 if $\mathrm{S}<\mathrm{K}$. Since there is no analytical solution for $\Delta_{t}^{G}$ the deltas are computed via Monte Carlo simulations.

\subsection{The Black-Scholes option pricing model}

The Black-Scholes theorem was first published in 1971 (Black and Scholes [1]). It is most widely used model for pricing model. The model states if $\mathrm{S}(\mathrm{t})$ is asset price that follows a generalised Wiener Process

$$
d S(t)=\mu S(t) d t+\sigma S(t) d z(t)
$$

$z(t)$ is a Brownian motion, the interest rate and volatility are constant. Then a call option on the asset, expiring at time $\mathrm{T}$ and with strike price $\mathrm{K}$ will have value at time $\mathrm{t}$ :

$$
C(t, T)=S(t) N\left(d_{1}\right)-K e^{-r(T-t)} N\left(d_{2}\right)
$$




$$
\begin{aligned}
& \text { where, } d_{1}=\frac{\ln \left(\frac{S(t)}{K}\right)+\left(r+\frac{\sigma^{2}}{2}\right)(T-t)}{\sigma \sqrt{T-t}} \\
& d_{2}=\frac{\ln \left(\frac{S(t)}{K}\right)+\left(r-\frac{\sigma^{2}}{2}\right)(T-t)}{\sigma \sqrt{T-t}} \\
& N(x)=(2 \pi)^{-1 / 2} \int_{-\infty}^{x} \exp \left(-\frac{z^{2}}{2}\right) d z
\end{aligned}
$$

$\mathrm{N}(\mathrm{x})$ is the cumulative probability distribution for a standard normally distributed variable. The Black-Scholes delta is given by $\mathrm{N}(\mathrm{d} 1)$. Which measures the sensitivity to the underlying instrument.

\subsubsection{Implied volatility}

The implied volatility is volatility parameter, which equates the market price with the price given by the Black-Scholes formula. The implied volatility, $\sigma_{t}^{B S}(K, T)$ is a function of $\mathrm{K}$ (Strike) and T (time to maturity) (Hull [19]). The two most interesting features of the volatility surface, which have been studied and analysed by researchers, are, the volatility smile (skew) and the term structure and the level of implied volatility changes with time. The volatility smile is a key indicator of unrealistic assumption of constant volatility. Whereas the changes in the implied volatility level with time is seen by the deformation of the volatility surface with time. Therefore, the ability to capture the deformation of the volatility surface will lead to accurate option pricing.

Cont and da Fonseca [20] have expressed mathematically the implied volatility surface as follows:

$$
C_{B S}\left(S_{t}, K, \tau, \sigma_{t}^{B S}(K, T)\right)=C_{t}^{*}(K, T)
$$

where $\sigma_{t}^{B S}(K, T)>0$

The value of the call option as a function of the volatility is a monotonic mapping from $\left[0,+\infty[\right.$ to $] 0, \mathrm{~S}_{\mathrm{t}}-\mathrm{Ke}^{-\mathrm{rt}}\left[\right.$. The implied volatility $\sigma_{t}^{B S}(K, T)$ of a call option with strike $\mathrm{K}$ and price maturity of $\mathrm{T}$ is dependent on $\mathrm{K}$ and $\mathrm{T}$. If $\mathrm{K}$ and $\mathrm{T}$ are fixed, $\sigma_{t}^{B S}(K, T)$ can be generalised to follow a stochastic process. For a fixed $t$ the value will depend on the options characteristics such as maturity and strike level K. Eqn. (17) represents the volatility surface at time t.

$$
\begin{aligned}
& \sigma_{t}^{B S}:(K, T) \longrightarrow \sigma_{t}^{B S}(K, T) \\
& I_{t}(m, \tau)=\sigma_{t}^{B S}(m S(t), t+\tau)
\end{aligned}
$$

where $\mathrm{m}$ is the moneyness, $I_{t}(m, \tau)$ is the implied volatility function

The two most important features of this surface are volatility term structure (or volatility smile) and the changes in the volatility levels with time. Thus the evolution in time of this surface will reflect the evolution of market option prices. 


\subsection{Artificial neural network (ANN)}

The ANN consists mainly of an input layer, one more hidden layers and an output layer. The layers are connected via a set off weights. The hidden layer and the output layer consist of individual neurons. The inputs are multiplied by the weights and a bias term is added which then constitutes the input to the activation function. This is then served as the inputs to the proceeding layer. The activation of the output layer is given by,

$$
F(x)=G\left(\sum_{J} w_{i j} H\left(\sum_{K} w_{j k} x_{k}+B_{k}\right)+B_{J}\right)
$$

The activation functions of the neurons could be chosen to be linear or nonlinear functions. A sum of error-squared function is normally used as the objective function for the training the MLP. So the MLP is trained to minimize this function with respect to the in-sample data.

$$
E=\frac{1}{N} \sum_{1}^{N}\left(t_{i}-F_{i}(x)\right)^{2}
$$

The MLP performance is dependent on the initial values of the weights. To overcome this issue, the neural network is trained 50 times using different initial values for the weights. The weight set that introduces the least error is then adopted.

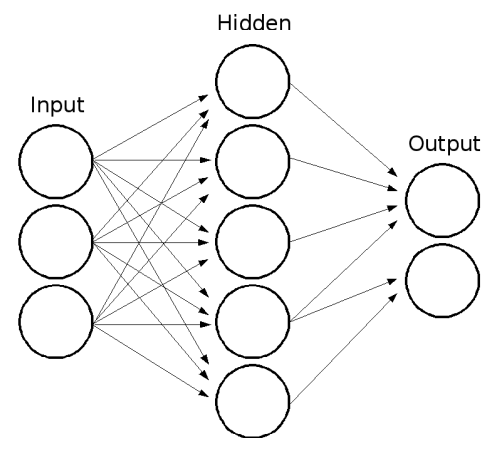

Figure 1: $\quad$ Single layer artificial neural network.

\section{The data}

The data used in this research consists of European call options on the FTSE 100 index traded at the London International Financial and Options Exchange (LIFFE). The data obtained in this research was obtained from SIRCA (http://www.sirca.org.au/), which covered a two-year period starting from $2 / 1 / 2000$ and ends on 31/12/2001. The sample made up of 63,094 call options and the daily Index value (adjusted for dividends). 
The following constraints were applied to filter the data series, Moneyness (Index/Strike) outside [-1.01,0.9], maturity greater 175 days and less than 7 days and Close price less than five where removed. The data series was then reduced to 26533 options. GB Libor rates were used for the risk free rate inputs to the models.

The parameters for the GOPM and Black-Scholes were estimated on a daily basis. However, when optimising the ANN the data was split into 3 sets: insample, validation and out-of-sample. The in-sample set is used as inputs to the ANN thus impacting the weights. The validation set is used to evaluate the error at each epoch. The training of the network is terminated when the error of the validation increases. The out-of-sampled is data set is used for evaluating the ANN model. The hedging analysis was done over 1 month horizon for the month of Feburary-2000.

\section{Experimental design}

The aim of this research is to investigate the capability of Neural Networks to capture the market volatility dynamics, and hence accurately price call options. The competing models are the GOPM; two Neural Networks trained on the option price (NNp) and implied volatility (NNiv) respectively. The models are then compared based on the out of sample pricing accuracy and the hedging performance.

\subsection{GOPM parameters}

The GOPM parameters were estimated for each day of the data sample. This was achieved by minimizing the average sum squared error eqn. (21) (Aboura [21], Lehnert [22]) over all options on day $t$, with parameters form day $t-1$ as the initial values (Lehar et al. [23]).

$$
\operatorname{Min} \operatorname{SSE}(\Theta)=\sum_{t=1}^{T} \sum_{n=1}^{N}\left(\frac{\left(\hat{C_{t, n}}-C_{t, n}\right)}{C_{t, n}}\right)
$$

where $\Theta=\left\{\beta_{0}, \beta_{1}, \beta_{2}, \lambda\right\}$

Where $C$ and $C$ are the model theoretical price and the actual call price respectively. The number of Monte Carlo simulation was initially set to 15,000 . This was sufficient to generate stable estimates. In some case the number of simulation had to be increased to 30,000 .

\subsection{ANN models and training}

ANN has been applied to many option pricing problems. Most researchers focus on training the ANN to estimate the option price directly (Donaldson and Kamstra [24], Meissner and Kawano [13] and Hutchinson et al. [25]). In this research, two methods are examined; the first method is commonly used in research where the neural network is training on the option price. The second 
method the neural network is trained using the Black-Scholes implied volatility as the target output. This method is similar to the one adopted by Duan [26]. The neural network was optimized by varying the number of inputs, length of in sample and validation sets, and the number of hidden unites. The combination that gives the lowest error is chosen. The weight values were initialized randomly. To eliminate the dependency on the initial weight values this process was repeated 50 times.

\subsubsection{ANN with pricing options}

This approach has been widely studied in literature. This approach was also followed by Bennell and Sutcliffe [17]. The input data was partitioned according the moneyness of the options in-the money (ITM), at-the-money (ATM) and outof-the-money (OTM). For each set, a different network was used. This method has been proven to improve the pricing capabilities of the ANN (Yao et al. [15]). The in-sample and validation set for ATM and ITM were 168 days and 84 respectively. For OTM 64 and 20 days for the in-sample and validation set were used.

The homogeneity hint (Merton [27]) is used to simplify the input parameters (Garcia and Cirano [28]). The Strike and Index can be combined into a single input Index/Strike that is also interpreted as the measure of the option's moneyness. The other inputs used are the option maturity and historical volatility. The target set is the Close price/ Strike. From this point onwards this models will be referred to as NNp.

\subsubsection{ANN with implied volatility}

In this paper adopt a new methodology is adopted which will allow the neural network to capture the dynamics of the underlying instruments and the changes in the volatility levels through time. To achieve the desired effect the neural network is trained on the Black-Scholes implied volatility. In this exercise, the data set was not partitioned by moneyness, therefore allowing the neural network full visibility of the volatility surface. This approach is a mapping exercise described in eqn. (17).

After experimentation with the different inputs, the following combination was selected for optimal performance. The in sample and validation set were 20 and 5 days respectively, and the output sample was 1 day. The inputs used were moneyness (index/strike), Time to expiry and historical volatility. The target data set was the Black-Scholes implied volatility. Once the training of the ANN is completed, the implied volatility produced by the ANN would be plugged into the Black-Schole formula to obtain an analytical price of the option. The same concept is applied to generating the delta's, where the Black-Scholes delta is used with the implied volatility produced via the neural network. This model would be referred to as NNiv.

\section{Performance measure}

The first measure of the model performance is its ability to price options out of sample. However, this measure does not give a clear indication of the models 
ability to generate profits. Therefore, the hedging performance of the model is a much better indication of the models ability to capture the underlying dynamics in the market.

\subsection{Pricing accuracy}

To analyze the models performance, Relative Pricing Error (PE) and Absolute Relative Pricing Error (APE) were calculated for each model.

$$
\begin{aligned}
& R P E=\frac{1}{n} \sum_{i=1}^{n}\left(\frac{\hat{C}_{i}-C_{i}}{C_{i}}\right) \\
& A R P E=\frac{1}{n} \sum_{i=1}^{n}\left|\frac{\hat{C}_{i}-C_{i}}{C_{i}}\right|
\end{aligned}
$$

where $C$ and $C$ is the model theoretical call price and the actual call price. The errors are reported against maturity and Moneyness.

\subsection{Delta hedging}

Most papers consider a hedge portfolio with one unit short in an option, $\Delta$ units of the underlying and the rest in a risk free asset (Schittenkopf and Dorffner [29]). In this research a hedge portfolio is constructed in a similar fashion to that adopted by Amilon [14] and Vahamaa [30]. That is we buy that are under priced (where theoretical option price is higher then market price) and sell the options that are overpriced. For under priced options we construct the following portfolio:

We by option $V_{0}^{C}=C_{0}$ and sell index $V_{0}^{I}=-I_{0} \Delta_{0}$ and put the rest in riskfree asset, $V_{0}^{B}=I_{0} \Delta_{0}-V_{0}^{C}$. The portfolio is then replicated as follows:

$$
\begin{gathered}
V_{t}^{I}=-I_{t} \Delta_{t} \quad, \quad V_{t}^{C}=C_{t} \\
V_{t}^{B}=\exp (r t) V_{t-1}^{B}+I_{t}\left(\Delta_{t}-\Delta_{t-1}\right)
\end{gathered}
$$

The value of the portfolio at time $\mathrm{T}$ is given by,

$$
V_{T}=V_{T}^{B}+V_{T}^{I}+V_{T}^{C}
$$

Which gives us the absolute hedging error

$$
\xi=\exp (r t)\left|V_{T}\right|
$$

Where, $V_{t}^{B}, V_{t}^{I}$ and $V_{t}^{C}$ are the amount invested in the risk free asset, index and call option at time $\mathrm{t}$.

\section{Results}

Below are the estimated mean values of the GOPM parameters. 
The risk premium is extracted from the option prices rather than from the index time series. It was found to be a little weak which intern will have minor impact on the option price. The Annual volatility was found to be approx $22.37 \%$ which is a close approximation to the historical volatility when estimated on the index prices $20.7 \%$.

Table 1: $\quad$ GOPM average parameters.

\begin{tabular}{|c|c|c|c|c|}
\hline$\beta_{0}$ & $\beta_{1}$ & $\beta_{2}$ & $\gamma$ & $\sqrt{\sigma}$ \\
\hline $3.109 \mathrm{E}-05$ & $1.848 \mathrm{E}-01$ & $3.184 \mathrm{E}-01$ & $6.745 \mathrm{E}-01$ & $22.37 \%$ \\
\hline
\end{tabular}

Table 2: $\quad$ Relative pricing errors for all models.

\begin{tabular}{|c|c|c|c|c|c|}
\hline \multirow[b]{2}{*}{ Moneyness } & \multirow[b]{2}{*}{ Model } & \multicolumn{4}{|c|}{ Time to Maturity } \\
\hline & & LT & MT & ST & TOTAL \\
\hline \multirow[t]{4}{*}{ DOTM } & GOPM & 0.021 & -0.010 & -0.020 & -0.009 \\
\hline & NNp & -0.062 & -0.047 & 0.837 & 0.128 \\
\hline & BLACK-SCHOLES & -0.098 & -0.011 & 0.038 & -0.005 \\
\hline & NNiv & 0.034 & 0.114 & -0.024 & 0.073 \\
\hline \multirow[t]{4}{*}{ OTM } & GOPM & 0.027 & -0.007 & 0.059 & 0.024 \\
\hline & NNp & -0.145 & -0.365 & 0.269 & -0.085 \\
\hline & BLACK-SCHOLES & -0.497 & -0.691 & -0.901 & -0.762 \\
\hline & NNiv & 0.005 & 0.014 & 0.024 & 0.017 \\
\hline \multirow[t]{4}{*}{ ATM } & GOPM & 0.039 & 0.008 & 0.030 & 0.021 \\
\hline & NNp & -0.133 & -0.083 & 0.238 & 0.044 \\
\hline & BLACK-SCHOLES & -0.272 & -0.240 & -0.215 & -0.232 \\
\hline & NNiv & 0.025 & 0.004 & 0.007 & 0.007 \\
\hline \multirow[t]{4}{*}{ ITM } & GOPM & 0.059 & 0.013 & -0.008 & 0.007 \\
\hline & NNp & 0.063 & -0.013 & 0.142 & 0.066 \\
\hline & BLACK-SCHOLES & -0.165 & -0.084 & 0.002 & -0.049 \\
\hline & NNiv & 0.040 & 0.008 & -0.019 & -0.002 \\
\hline \multirow[t]{4}{*}{ DITM } & GOPM & 0.069 & 0.059 & 0.044 & 0.057 \\
\hline & NNp & -0.088 & -0.104 & 0.010 & -0.051 \\
\hline & BLACK-SCHOLES & -0.098 & -0.011 & 0.038 & -0.005 \\
\hline & NNiv & 0.075 & -0.002 & -0.020 & 0.004 \\
\hline \multirow[t]{4}{*}{ All } & GOPM & 0.045 & 0.018 & 0.042 & 0.030 \\
\hline & NNp & -0.109 & -0.199 & 0.350 & 0.004 \\
\hline & BLACK-SCHOLES & -0.584 & -0.900 & -0.641 & -0.767 \\
\hline & NNiv & 0.022 & 0.043 & 0.009 & 0.027 \\
\hline
\end{tabular}


The performance measures used for all models are relative pricing error (RPE) and the absolute relative pricing error (ARPE). A positive or negative value of $\mathrm{PE}$ indicates overpricing or under pricing of the model respectively. Table (2) shows the RPE for all models. On average, the GOPM and the NNiv overprice call options, whereas the Black-Scholes and NNp under price the options. The NNp tends to over price ST options and under price options in the LT and MT range. The GOPM and NNiv have similar behavior where both models over price options over all maturities. The BLACK-SCHOLES model seems to under prices options over all maturities. In addition, it has the worst miss-pricing performance across all maturity and moneyness.

Table 3: $\quad$ Absolute relative pricing errors for all models.

\begin{tabular}{|c|c|cccc|}
\hline \multirow{2}{*}{ Moneyness } & \multirow{2}{*}{ Model } & \multicolumn{4}{|c|}{ Time to Maturity } \\
\cline { 3 - 6 } & & LT & MT & ST & TOTAL \\
\hline \multirow{2}{*}{ DOTM } & GOPM & 0.179 & 0.246 & 0.384 & 0.267 \\
& NNp & 0.590 & 0.513 & 1.014 & 0.625 \\
& BLACK-SCHOLES & 0.993 & 1.815 & 1.289 & 1.584 \\
& NNiv & 0.167 & 0.269 & 0.358 & 0.273 \\
\hline OTM & GOPM & 0.105 & 0.157 & 0.272 & 0.202 \\
& NNp & 0.519 & 0.483 & 0.711 & 0.580 \\
& BLACK-SCHOLES & 0.502 & 0.713 & 0.984 & 0.809 \\
& NNiv & 0.102 & 0.154 & 0.262 & 0.195 \\
\hline \multirow{2}{*}{ ATM } & GOPM & 0.078 & 0.100 & 0.139 & 0.116 \\
& NNp & 0.263 & 0.212 & 0.400 & 0.295 \\
& BLACK-SCHOLES & 0.274 & 0.262 & 0.277 & 0.270 \\
& NNiv & 0.086 & 0.094 & 0.135 & 0.112 \\
\hline ITM & GOPM & 0.082 & 0.074 & 0.079 & 0.077 \\
& NNp & 0.154 & 0.131 & 0.232 & 0.181 \\
& BLACK-SCHOLES & 0.169 & 0.120 & 0.077 & 0.104 \\
& NNiv & 0.081 & 0.071 & 0.075 & 0.074 \\
\hline \multirow{2}{*}{ DITM } & GOPM & 0.039 & 0.061 & 0.050 & 0.053 \\
& NNp & 0.160 & 0.143 & 0.151 & 0.149 \\
& BLACK-SCHOLES & 0.099 & 0.074 & 0.051 & 0.069 \\
& NNiv & 0.098 & 0.066 & 0.058 & 0.068 \\
\hline \hline \multirow{2}{*}{ All } & GOPM & 0.121 & 0.167 & 0.233 & 0.188 \\
& NNp & 0.491 & 0.440 & 0.674 & 0.528 \\
& BLACK-SCHOLES & 0.589 & 0.924 & 0.742 & 0.818 \\
& NNiv & 0.119 & 0.172 & 0.222 & 0.185 \\
\hline
\end{tabular}


The GOPM and NNiv models over prices LT options across all moneyness. The worst miss pricing performance of the GOPM model has a RPE of 0.069 for LT-DITM contracts. This behavior is also seen for NNiv, where the model has the worst miss pricing for LT-DITM contracts with a RPE of 0.075. For BLACK-SCHOLES model the worst performance for ST-OTM options with a RPE of -0.901 and the NNp has a PE of 0.837 for ST-DOTM contracts.

The APE figures are displayed in table (3). On average the performance of GOPM and NNiv models increase with maturity. Again, the BLACK-SCHOLES model performs the worst on all accounts. On average, the NNiv has a slightly better performance than the GOPM. The GOPM model slightly performs better for MT contracts and the NNiv has a better performance for ST and LT contracts. All models have performed the worst for ST-DOTM contracts. In addition, the GOPM performs the best for LT-DITM whereas the NNiv and BLACK-SCHOLES are at their best for ST-DITM contracts. The NNiv seem to perform best for the MT-ITM contracts.

Table 4: $\quad$ Discounted absolute hedging error.

\begin{tabular}{|c|c|c|c|c|c|}
\hline & Model & LT & MT & ST & Total \\
\hline \multirow{3}{*}{ ATM } & NNiv & 0.38 & 19.56 & 15.66 & 15.20 \\
\hline & $\begin{array}{l}\text { BLACK- } \\
\text { SCHOLES }\end{array}$ & 0.29 & 19.09 & 15.74 & 15.06 \\
\hline & GOPM & 0.41 & 22.38 & 16.33 & 16.56 \\
\hline \multirow{3}{*}{ ITM } & NNiv & 0.41 & 23.37 & 27.47 & 22.83 \\
\hline & $\begin{array}{l}\text { BLACK- } \\
\text { SCHOLES }\end{array}$ & 0.31 & 21.99 & 29.65 & 23.22 \\
\hline & GOPM & 0.42 & 25.93 & 30.62 & 25.39 \\
\hline \multirow{3}{*}{ OTM } & NNiv & 0.23 & 9.91 & 6.70 & 7.57 \\
\hline & $\begin{array}{l}\text { BLACK- } \\
\text { SCHOLES }\end{array}$ & 0.20 & 9.90 & 5.89 & 7.28 \\
\hline & GOPM & 0.30 & 12.20 & 6.52 & 8.72 \\
\hline \multicolumn{2}{|c|}{ Total Nniv } & 0.33 & 16.67 & 17.03 & 14.90 \\
\hline \multicolumn{2}{|c|}{$\begin{array}{l}\text { Total } \\
\text { SCHOLES }\end{array}$} & 0.26 & 16.11 & 17.55 & 14.87 \\
\hline \multicolumn{2}{|c|}{ Total GOPM } & 0.37 & 19.18 & 18.27 & 16.56 \\
\hline
\end{tabular}

NNp will be excluded from the hedging analysis due to the unsatisfactory performance with the option pricing. The rest of the models are compared over 1 month horizon, where delta hedging was performed until the option expiry. Then absolute hedging error eqn. (25) was used as the benchmark statistic for all models. As shown in table (4) the BLACK-SCHOLES and NNiv on average are very close with a difference of 0.03 . But the NNiv on average performed better for the ITM options. The GOPM has the worst performance on all accounts. For ATM-ST and ITM-ST options, the NNiv performed better to the reset of the models. In addition, NNiv seem to get very close to the Black-Scholes. For 
instance, the OTM-MT option, the BLACK-SCHOLES and NNiv were in fact very close with a difference of 0.01 . However, the GOPM display far worse performance with big differences when compared to the Black-Scholes. The NNiv was clearly out performed the GOPM on all accounts expected for OTMST but with a difference of 0.18 .

\section{Summary and conclusion}

In this research, a new method for pricing options via neural networks is introduced. The neural network was trained using the implied volatility as the target output of the neural network rather then the options price. This method has shown to improve the out-of-sample pricing performance of the neural network. The neural networks performance was also compared to the GOPM and the Black-Scholes. The overall performance of the NNiv was slightly better than GOPM and much better than the Black-Scholes. The improvement in the neural network pricing performance is a result of the neural network ability to capture the underlying volatility structure and volatility levels with time. Which is apparent in its out of sample pricing abilities.

The models were also tested with respect to their hedging performance. The GOPM performed the worst. However, the Black-Scholes on averaged outperformed the others except for the ITM options where NNiv did better. The NNiv did perform better then the Black-Scholes in some categories and in most cases was trailing very closely. However, it did outperform the GOPM. There are a couple of factors that may have affected the outcome of the results. Firstly, the exclusion of options based on moneyness and maturity could have resulted in the loss of significant information. Also, the hedging strategy of keeping the option till maturity could skew the results. The method described in Amilon [14] would have been a better option. In addition, the use of the discounted absolute hedging error alone does not provide enough statistics to evaluate the model performance.

The results presented in this paper are very promising. It has been demonstrated the superiority of the neural networks over the GOPM. In addition, the ability of the neural network to produce an option price instantaneously enables it to be deployed as real time trading tool. An ongoing direction of this research is to extend the current findings to train the neural network on the Black-Scholes delta rather than using the neural networks implied volatility and to examine different types of neural networks such as RBF that may produce improved results.

\section{References}

[1] F. Black, and M. Scholes, The Pricing of Options and Corporate Liabilities. The Journal of Political Economy 81 (1973) 637-654.

[2] V. Henderson, The Black Scholes Model.

[3] P. Ritchken, and R. Trevor, Pricing Options under Generalized GARCH and Stochastic Volatility Processes. The Journal of Finance 54 (1999) 377402. 
[4] C. Peter, and J. Kris, Which GARCH Model for Option Valuation? Manage. Sci. 50 (2004) 1204-1221.

[5] R.F. Engle, and C. Mustafa, Implied ARCH Models from Options Prices. Journal of Econometrics 52 (1992) 2.

[6] J.C. Duan, The GARCH Option Pricing Model. Mathematical Finance 5 (1995) 13-32.

[7] S.L. Heston, S. Nandi, and A. Federal Reserve Bank of, A Closed-form GARCH Option Pricing Model, Federal Reserve Bank of Atlanta, 1997.

[8] T.I.M. Bollerslev, Generalized Autoregressive Conditional Heteroskedasticity. ARCH: Selected Readings (1995).

[9] J.C. Duan, and J.G. Simonato, Empirical Martingale Simulation for Asset Prices. Management Science 44 (1998) 1218-1233.

[10] O. Karaali, W. Edelberg, J. Higgins, M. Inc, and I.L. Schaumburg, Modelling volatility derivatives using neural networks. Computational Intelligence for Financial Engineering (CIFEr), 1997., Proceedings of the IEEE/IAFE 1997 (1997) 280-286.

[11] C. Schittenkopf, G. Dorffner, and E.J. Dockner, Forecasting timedependent conditional densities: a semi non-parametric neural network approach. Journal of Forecasting 19 (2000) 355-374.

[12] I. Kaastra, and M. Boyd, Designing a neural network for forecasting financial and economic time series. Neurocomputing 10 (1996) 215-236.

[13] G. Meissner, and N. Kawano, Capturing the Volatility Smile of Options on High-Tech Stocks-A Combined GARCH-Neural Network Approach. Journal of Economics and Finance 25 (2001) 276-292.

[14] H. Amilon, A neural network versus Black-Scholes: a comparison of pricing and hedging performances. Journal of Forecasting 22 (2003) 317335.

[15] J. Yao, Y. Li, and C.L. Tan, Option price forecasting using neural networks. Omega 28 (2000) 455-466.

[16] P. Tino, C. Schittenkopf, and G. Dorffner, Financial volatility trading using recurrent neural networks. Neural Networks, IEEE Transactions on 12 (2001) 865-874.

[17] J.A. Bennell, and C. Sutcliffe, Black-Scholes Versus Artificial Neural Networks in Pricing FTSE 100 Options, Dept. of Accounting and Management Science, University of Southampton, 2000.

[18] M. Hanke, Neural Network Approximation of Option Pricing Formulas for Analytically Intractable Option Pricing Models. Journal of Computational Intelligence in Finance 5 (1997) 20-27.

[19] J. Hull, Options, futures, and other derivatives, Prentice Hall Upper Saddle River, NJ, 2003.

[20] R. Cont, and J. da Fonseca, Deformation of implied volatility surfaces: an empirical analysis. Empirical Approaches to Financial Fluctuations (2001).

[21] S. Aboura, GARCH Option Pricing Under Skew. Journal: The ICFAI Journal of Applied Economics November 4 (2005) 78-86.

[22] T. Lehnert, Mandelbrot and the Smile. 
[23] A. Lehar, M. Scheicher, and C. Schittenkopf, GARCH vs. stochastic volatility: Option pricing and risk management. Journal of Banking and Finance 26 (2002) 323-345.

[24] R.G. Donaldson, and M. Kamstra, Forecast combining with neural networks. Journal of Forecasting 15 (1996) 49-61.

[25] J.M. Hutchinson, A.W. Lo, and T. Poggio, A Nonparametric Approach to Pricing and Hedging Derivative Securities Via Learning Networks. The Journal of Finance 49 (1994) 851-889.

[26] J.C. Duan, Cracking the smile. Risk 9 (1996) 55-59.

[27] R.C. Merton, Theory of Rational Option Pricing. The Bell Journal of Economics and Management Science 4 (1973) 141-183.

[28] R. Garcia, and Cirano, Pricing and Hedging Derivative Securities with Neural Networks and a Homogeneity Hint, CIRANO, 1998.

[29] C. Schittenkopf, and G. Dorffner, Risk-neutral density extraction from option prices: improved pricing with mixture density networks. Neural Networks, IEEE Transactions on 12 (2001) 716-725.

[30] S. Vahamaa, Delta Hedging with the Smile. Financial Markets and Portfolio Management 18 (2004) 241-255. 